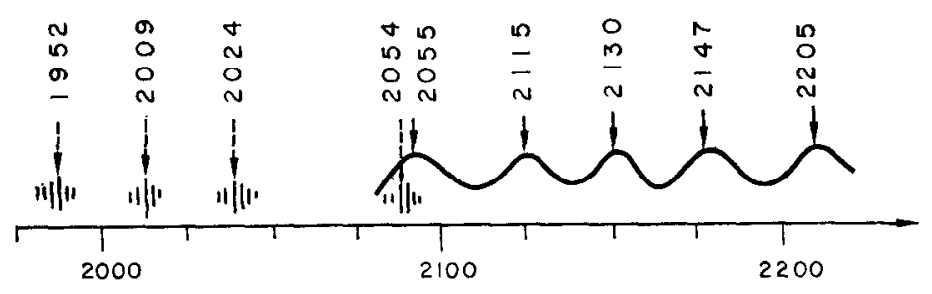

NZSLT

FIg. 2. A SCHEMATIC COMPARISON BETWEEN THE 'QUASI-PERIODIC EMISSION' EVENT OF 4 MAY 1967 AND A LONG PERTOD PC RECORDED IMMEDIATELY AFTERWARDS. THE EMISSION WAS RECORDED AT DUNEDIN WHLST THE pC WAS RECORDED $\sim 150 \mathrm{~km}$ SOUTH-WEST OF DUNEDIN.

The $15 \mathrm{~min}$ pc shown in Fig. 2 is then probably a ground-level manifestation of the geotail oscillations which had previously produced the plasma bunches responsible for the 'quasi-periodic emission'.

Thus it appears that the micropulsation 'quasi-periodic emission' under discussion can be adequately explained; though it is indeed a pity that these interesting events are not as common as their VLF counterparts.

Department of Physics

R. J. OFFEN

University of Otago,

Dunedin,

New Zealand.

\title{
REFERENCES
}

Helliweli, R. A. (1965). Whistlers and Related lonospheric Phenomena (Stanford University Press).

HelurWelL, R. A. (1967). J. geophys. Res. 72, 4773.

KokUBun, S. (1970). Rept. Ionosph. Space Res. Japan 24, 24.

Jacobs, J. A. and Watanabe, T. (1967). Planet. Space Sci. 15, 799.

Kennel, C. F. and Petschek, H. E., AVCO Res. Rep. No. 259, 1966.

LIRMOHN, H. B. (1967). J. geophys. Res. 72, 39.

MCClaY, J. F. and RADOsKI, H. R. (1967). J. geophys. Res. 72, 4525.

OFFEN, R. J. (1970). High Frequency Geomagnetic Micropulsations, Ph.D. Thesis, Otago University.

Patel, V. L. (1968a). Physics Letts 26, 596.

PATEL, V. L. (1968b). J. geophys. Res. 73, 3407.

Poletri, M. J. (1968). Private communication.

SisCOE, G. L. (1969). J. geophys. Res. 74, 6482.

Planet. Space Sci. 1972, Vol. 20, pp. 1787-1790. Pergamon Press. Printed in Northern Ireland

\section{COLLISIONLESS PLASMA FLOW OVER A CONDUCTING SPHERE}

\section{(Received 10 March 1972)}

The complexity of phenomena involved in the interaction between a spacecraft and its environmental space plasma are well recognized. Attempts to study aspects of these phenomena and effects were undertaken in the last decade mainly from the theoretical point of view (a detailed review including an extensive bibliography is given by Gurevich $e t$ al. $\left.{ }^{(1)}\right)$ However, the present situation is still controversial in various respects. There is some doubt as to the relative importance of various principal plasma parameters in defining the characteristics of the disturbed zone around satellites, and there is also some controversy as to the significance and reality of assumptions, both physical and mathematical, used in the numerous theories. Attempts to study the disturbed zone via in situ observations were also made by Samir ${ }^{(2)}$ but these observations are a priori bound to be fragmentary in nature. No space mission has ever been launched with wake studies as the principal experimental objective. The available in situ observations consist of studies made by using different plasma probes namely, planar and cylindrical Langmuir probes and multigrid retarding potential analyzer. The data was obtained from satellites of a variety of shapes and sizes which 
were launched at different times during the 1960's and into orbits with different perigee/apogee altitudes. Despite these difficulties, the available in situ observations are very valuable and were used partly to examine various theories. ${ }^{(1,3)}$

With the possible experimental openings in future Space Shuttle/Skylab/Space Station missions to be launched in the late 1970's and 1980's, the scientifically fundamental problem of the characteristics of the zone of disturbance around a spacecraft has been renewed and is now of growing interest.

It, therefore, becomes clear that laboratory simulation (important and of scientific interest in their own right) should be considered as a complementary study to the future space effort. We have, therefore, embarked on an extensive program in which different aspects of spacecraft-space plasma interactions will be studied utilizing standard probes and at the same time engaging in the development of new methods and techniques. Laboratory simulation studies have been performed during the 1960's and Refs. (4-6) represent some of the more recent publications.

Our experimental studies are performed in a plasma wind tunnel at the Space Sciences Laboratory of Marshall Space Flight Center. ${ }^{(7)}$ At present, the above facility is able to investigate phenomena over the following range of conditions: drift velocity, $9-500 \mathrm{~km} / \mathrm{sec} ;$ density, $10^{2}-10^{4} \mathrm{~cm}^{-3} ; T_{e}, 700-30,000^{\circ} \mathrm{K}$. This range will be extended. The plasma source is a Kaufman-type ion thruster, which operates on $\mathrm{N}_{2}$ gas with an emissive tungsten wire neutralizer, and provides a steady plasma beam. Two dimensional mappings of the flow field have been obtained. It would not be impossible to obtain three dimensional mappings.

It is well known that the characteristics of the zone of disturbance created by the spacecraft motion depends on plasma parameters such as $R_{0} / \lambda_{D}, T_{0} / T_{+}$, eq $\phi_{e} / k T_{0}$ and $V_{s} / \sqrt{2 k T_{d} / M_{t}}$, where $R_{0}=$ radius of the spacecraft, $\lambda_{D}=$ the Debye length, $V_{s}=$ spacecraft velocity, $T$, and $T_{+}=$electron and ion temperatures, respectively, $M_{+}=$ionic mass, $\phi_{s}=$ spacecraft potential with respect to the ambient space plasma.

If the geomagnetic field is included, then parameters such as the ion and electron Larmor radii with respect to $R_{0}$ and $R_{p}$ (probe radius) should be considered. The structure of the disturbed zone also depends on the geometry, size, and surface materials of the spacecraft.

Single body satellites cannot, a priori, map the entire zone of disturbance behind the body. This is due to the fact that probes are mounted on the satellite surfaces and/or on small booms. A laboratory simulation study can, however, map the entire zone of disturbance behind the body.

An example showing some preliminary results of the collisionless flow pattern over a conducting sphere is presented in Fig. 1. This figure presents transverse ion profiles at various positions along the wake axis $\left(r / R_{0}\right)$ where $r$ is the distance along the wake axis downstream and $R_{0}=3 \mathrm{~cm}$. The data were taken with a shielded Faraday cup. The plasma parameters for these axial/transverse profiles are:

$$
\begin{gathered}
R_{0} / \lambda_{D}=0.8, \\
V_{s} / \sqrt{\frac{\sqrt{2 k T} e}{M_{+}}}=17, \\
\frac{T_{e}}{T_{+}} \gg 1,
\end{gathered}
$$

and

$$
\frac{e \phi_{s}}{k T_{e}} \simeq-5
$$

The closest distance of the ion probe to the sphere (downstream) in terms of normalized distance $\left(r / R_{0}\right)$ is $\simeq 0.07$. At this point, the 'valley' in the transverse profile shows no detectable positive current. Proceeding downstream, we observe the wake to become filled with ions. From $\left(r / R_{0}\right)=14.8$, an ion increase in the wake (on the axis) is clearly seen reaching its maximum at about $\left(r / R_{0}\right)=20$ and then splitting into two peaks which finally diffuse in the far-wake zone. The finite length of the tunnel did not allow the observation of the precise location of total diffusion into the ambient plasma.

A physical explanation to the flow behavior shown in Fig. 1 can be in terms of 'two converging ion streams'. This is of interest because of the low density and collisionless plasma regime in the tunnel. A similar case was treated theoretically in great detail by Taylor, ${ }^{(8)}$ and our results are in accord with Taylor's model. Interpreting our results in terms of two streams in a highly rarefied collisionless plasma has a bearing on flow concepts used in magnetospheric physics.

It is of interest to determine quantitatively the variation of the 'crossing point' zone of the two streams in the wake as a function of negative body potential $\left(\phi_{s}\right)$. An example of such a dependence is shown in Fig. 2. The range of $\phi_{8}$ covered in volts is $0.5 \leq \phi_{8} \leq 9 \cdot 0$. It is clearly seen that as the potential on the sphere becomes more negative with respect to the environmental plasma, the 'focusing' effect becomes stronger. The most significant part of the depleted-wake becomes shorter and converges toward the sphere. It is also seen from Fig. 2 that the effect of body potential on the two-stream focusing exhibits an exponential dependence. This is obviously not in agreement with the 'neutral approximation' often used in theoretical studies; i.e. assuming the ion motion to behave in a manner similar to the neutral particle motion. 


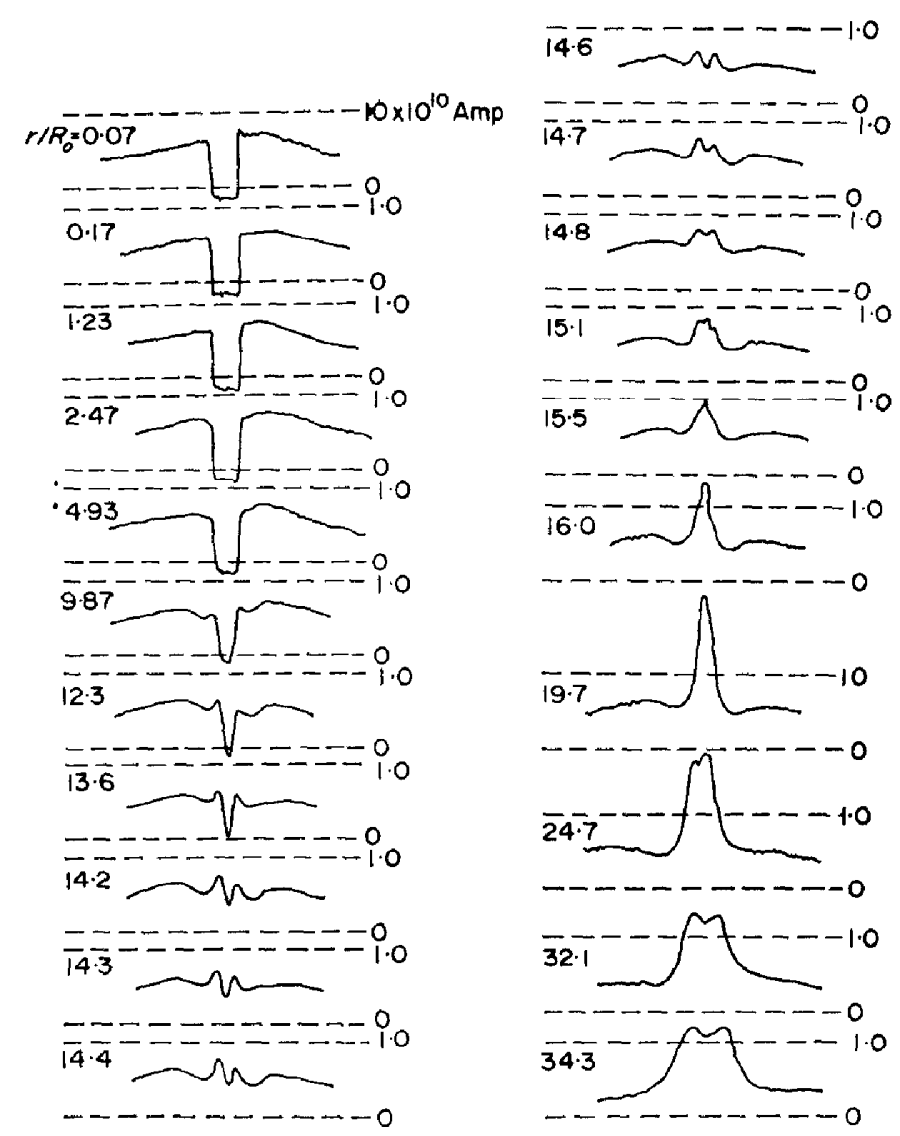

Fig. 1. Transverse ION PRofiles at various positions along the Wake axis. $\left(r / R_{0}\right)$ Is THE NORMALIZED DISTANCE DOWNSTREAM.

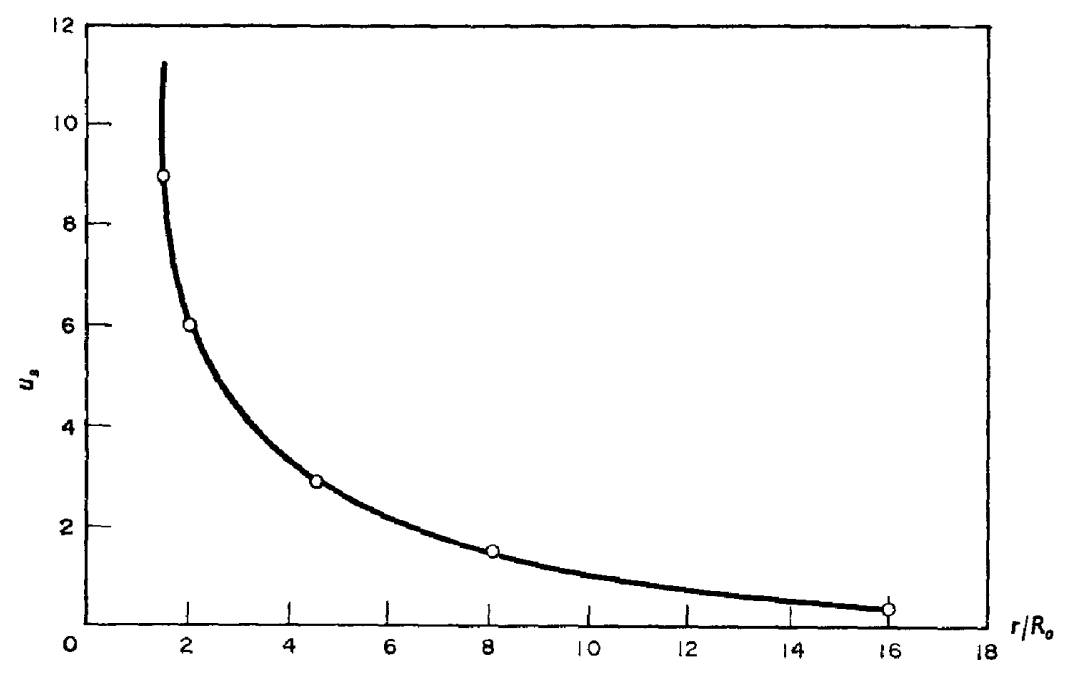

Fig. 2. VARIATION OF THE 'CROSSING-POINT' LOCATION IN THE WAKE IN TERMS OF ( $\left.r / R_{\downarrow}\right)$ with BODY POTENTIAL (IN VOLTS) RELATIVE TO GROUND $\left(u_{s}\right)$. 
Although this particular example does not simulate specifically the satellite-ionosphere interaction, it is relevant to highly hypersonic flows over rockets at altitudes above the $F 2$ region of the ionosphere. The conditions $e \phi_{a} / k T_{a} \approx-5$ is realistic and relevant to a variety of rapidly moving bodies in space, and $\left(R_{0} /\right.$ $\left.\lambda_{D}\right) \cong 1$ is realistic for probes mounted on booms onboard aeronomy satellites and for small vehicles. We do not know of any study which presents such a detailed structure especially in the region where the two streams converge.

Acknowledgements - We gratefully acknowledge the opportunity given to us by Mr. Gerhard B. Heller and Dr. Rudolf Decher of Space Sciences Laboratory, Marshall Space Flight Center, to perform experiments regarding spacecraft-space plasma interactions in the laboratory's tunnel. Thanks are due to Dr. E. R. Schmerling of NASA Headquarters for his interest and encouragement. We also acknowledge Mr. W. Roberts of MSFC for his support. One of us (U.S.) acknowledges support under Grants NGR 23-005-320 and NGR 23-005-151.

Space Sciences Laboratory,

Marshall Space Flight Center,

N. H. STONE

Huntsville, Alabama,

U.S.A.

Space Physics Research Laboratory,

University of Michigan, Ann Arbor, and

Department of Environmental Sciences,

Tel-Aviv University, Israel

W. A. Oran

URI SAMIR

\title{
REFERENCES
}

1. A. V. Gurevich, L. P. Pitaevskil and V. V. Smirnova, Space Sci. Rev. 9, 805 (1969).

2. U. SAMTR, Report ES-71-006, Dept. of Environmental Sciences, Tel-Aviv University, Israel (1971).

3. U. SAMTR and H. JEW, Electron density distribution in the near wake of an ionospheric satellite: A theory and experiment comparison. Submitted for publication in, J. Geophys. Res. (1972).

4. S. Lederman, M. H. BLOOM and G. F. Widhopf, AIAA J. 7, (8), 1421 (1969).

5. S. D. Hester and A. A. SonIN, Phys. Fluids 13, (3), 641 (1970).

6. I. A. Bogashchienko, A. V. Gurevich, R. A. Salimov and Yu. I. Eidelman, Soviet Physics, JEPT 32, (5), 841 (1971).

7. N. H. Stone and W. K. RehmanN, NASA TND-5894 (1970).

8. J. C. TAYLOR, Planet. Space Sci. 15, 155 \& 463 (1967).

\section{QUASIMONOCHROMATIC WHISTLER MODE PACKETS OF SLOWLY VARYING AMPLITUDE}

\author{
(Received 16 March 1972)
}

\begin{abstract}
A nonlinear interaction between the whistler mode wave packet and resonant particles is considered. An evolution of the packet's shape is investigated in detail.
\end{abstract}

Nonlinear effects produced by the interaction of whistlers with resonant particles have been discussed in a large number of papers (e.g. Roux and Solomon, 1970; Coriniti et al., 1971; Bud'ko et al., 1971). However, either packets with rather broad spectrum to which random phase approximation is applicable, or monochromatic plane waves of infinite length were considered. In the present paper we deal with whistler mode (W.M.) packets, which have such a narrow spectrum, that they cannot be treated in the random phase approximation. For experimental evidence for the significance of such cases see. e.g., Coroniti et al ${ }^{(2)}$ Our results are also applicable to VLF waves, radiated by transmitters and propagating along the geomagnetic field lines in the upper ionosphere and magnetosphere.

The numerical investigation of particle trajectories in W.M. packet was performed by Ashour-Abdalla.(4) We use an analytical asymptotic method which permits us to elucidate some new features of nonlinear evolution of the packet in stable and unstable plasmas. 